\title{
Early nonlinear regime of MHD internal modes: the resistive case
}

\author{
M.C. Firpo \\ Laboratoire de Physique et Technologie des Plasmas (C.N.R.S. UMR 7648), Ecole \\ Polytechnique, 91128 Palaiseau cedex, France
}

\begin{abstract}
It is shown that the critical layer analysis, involved in the linear theory of internal modes, can be extended continuously into the early nonlinear regime. For the $m=1$ resistive mode, the dynamical analysis involves two small parameters: the inverse of the magnetic Reynolds number $S$ and the $m=1$ mode amplitude $A$, that measures the amount of nonlinearities in the system. The location of the instantaneous critical layer and the dominant dynamical equations inside it are evaluated self-consistently, as $A$ increases and crosses some $S$-dependent thresholds. A special emphasis is put on the influence of the initial $q$-profile on the early nonlinear behavior. Predictions are given for a family of $q$-profiles, including the important low shear case, and shown to be consistent with recent experimental observations.
\end{abstract}

Key words:

Magnetohydrodynamics, nonlinear regime, internal modes, sawtooth oscillations PACS: 52.30.Cv, 52.35.Py, 52.35.Mw, 52.55.Tn

The $m=n=1$ internal modes, such that the safety factor goes below one for some inner radius, remain critical macroscopic modes for large scale tokamak plasma dynamics and confinement. They are particularly involved in sawtooth oscillations and crashes. This is a common deleterious phenomenon as conventional tokamak discharges eventually operate with $q_{0}<1$ since current density tends to a peaked profile. Additionally, the $m=n=1$ internal modes form a laboratory prototype for reconnection. Such phenomena typically proceed beyond linear regime.

We shall consider here the $m=n=1$ purely resistive mode [1] that is ideally marginally stable. The original motivation of this work was to understand the growth of the $m=1$ resistive mode up to its nonlinear saturation, on the basis of some striking numerical simulations performed by Aydemir [2] and previous observations [3]. Within the reduced MHD framework in cylindrical coordinates and some given $q$-profile [2], the time behavior of the kinetic energy in 
the $m=1$ mode amounts to an initial exponential growth consistent with the linear regime, followed by a transient stage where the growth rate decreases, that is brutally interrupted by a second exponential growth in the nonlinear regime. This second exponential stage eventually terminates, as the kinetic energy in the $m=1$ mode saturates which coincides with the completion of magnetic reconnection.

The reduced MHD system under consideration reads

$$
\begin{aligned}
& \frac{\partial U}{\partial t}=[\phi, U]+[J, \psi], \\
& \frac{\partial \psi}{\partial t}=[\phi, \psi]+\eta\left(J-J_{0}\right) .
\end{aligned}
$$

Helical symmetry is assumed: The poloidal and toroidal angles, respectively $\theta$ and $\varphi$, only come in through the helical angle $\alpha=\varphi-\theta . \phi$ and $\psi$ are the plasma velocity and helical magnetic field potentials: the velocity is $\mathbf{v}=\hat{\varphi} \times \nabla_{\perp} \phi$ and the magnetic field $\mathbf{B}=B_{0 \varphi} \hat{\varphi}+\hat{\varphi} \times \nabla_{\perp}\left(\psi-r^{2} / 2\right) . U=\nabla_{\perp}^{2} \phi$ is the vorticity and $J=\nabla_{\perp}^{2} \psi$ the helical current density, with $\nabla_{\perp}^{2} \equiv r^{-1} \partial_{r} r \partial_{r}+r^{-2} \partial_{\alpha}^{2}$. Poisson brackets are defined by $[\phi, U]=-\hat{\varphi} \cdot\left(\nabla_{\perp} \phi \times \nabla_{\perp} U\right)=r^{-1}\left(\partial_{r} \phi \partial_{\alpha} U-\right.$ $\left.\partial_{r} U \partial_{\alpha} \phi\right)$. Eqs. (1)-(2) are dimensionless: Time has been normalized to the poloidal Alfvén time, the radial variable $r$ to the minor radius, and $\eta$ is the inverse of the magnetic Reynolds number $S$, and is given by the ratio of the poloidal Alvén time to the resistive one. In high-temperature fusion plasmas, $\eta$ is typically much smaller than one.

Consider equilibria such that, for some internal radius $r_{s 0}<1, q\left(r_{s 0}\right)=1$, that is $\psi_{0}^{\prime}\left(r_{s 0}\right)=0$. Then, due to the Ohm's law (2), plasma volume divides in two region. Far from the $q=1$ surface (outer domain), plasma behaves ideally whereas, in the vicinity of $r_{s 0}$ (inner region), resistivity plays a crucial, destabilizing, role. Linear theory [1] uses asymptotic matching analysis to provide $m=1$ eigenfunctions in the form $A(t) f_{L}(r) \exp (i \alpha)$ valid in the whole domain. In the outer (ideal) domain, this solution is valid, that is nonlinear effects are negligible, as long as $A \ll 1$ [4]. Injecting the linear solutions $\psi_{1}(r, \alpha, t)=A(t) \psi_{L}(r) \exp (i \alpha)$ and $\phi_{1}(r, \alpha, t)=A(t) \phi_{L}(r) \exp (i \alpha)$ into (1)(2) calls for an amplitude expansion. The procedure has been given in Refs. $[4,5]$. The particularity of the linear radial eigenfunctions $\psi_{L}(r)$ and $\phi_{L}(r)$, that needs a careful consideration, is that they have strong gradients inside the critical layer. More precisely, their radial derivatives are of the order of the inverse of the critical layer width, that is $\mathcal{O}\left(\eta^{-1 / 3}\right)$. This means in particular that this approach restricts to situations strictly above marginal stability and where the linear regime is well defined, with clear scalings, yielding the resistive ordering, and non-pathological $q$-profiles (in the sense of Ref. [6]).

We wish then to answer the question: "How does the $m=1$ resistive mode 
develop into the nonlinear regime ?" To do this, let us first recognize that the problem involves two small parameters. An obvious one is the resistivity $\eta$. However, considering it to be the only one small parameter, in some perturbation analysis with conventional expansions of the type $f=f_{0}+\eta f_{1}+\ldots$ would lead to a dead end: this would bring up a singular expansion, with additional $\eta \ln (\eta)$ terms, with no asymptotic validity unless assuming that the mode amplitude is always kept vanishingly small. It is interesting to note that such a procedure would actually be valid for the tearing mode with the small parameter limit $\Delta^{\prime}[7,8]$. In the present case, such a perturbation analysis would be ill-posed. A second small parameter enters the game, the $m=1$ mode amplitude $A$ that can be viewed as an indicator of the amount of nonlinearities in the system. As previously said, the approach will then be that of an amplitude expansion.

The first step will be to determine the end of validity of the linear regime. In the outer domain, this occurs for $A$ of order one [4] but, in the inner domain, the linear solution breaks earlier. This occurs when mode coupling terms such as $\left[\phi_{1}, U_{1}\right]$ becomes of the same order order as linear terms, that is for $A \gtrsim \eta^{2 / 3}$. At this point, $m=0$ and $m=2$ components begin to be "fed" nonlinearly by mode coupling terms: the $m=0$ and $m=2$ modes are nonlinearly driven. However, these mode coupling terms, quadratic in $A$, do not affect the $m=1$ dynamics so that one could say that the $m=1$ mode is still linear. At this stage, it is easy to check that the dominant equations on the $m=1$ component are still the linear ones. This means that the radial structure of the solution should remain close to the linear one. Given that, it is possible to include the correction to the linear theory due to the new location of the critical layer. Because of the $m=1$ perturbation, the critical layer is not expected to remain fixed at $r_{s 0}$. The real helical magnetic field potential inside the critical layer (for $\left|r-r_{s 0}\right| \lesssim \eta^{1 / 3}$ ) is

$$
\psi(r, \alpha, t)=\frac{1}{2} \psi^{\prime \prime}\left(r_{s 0}\right)\left(r-r_{s 0}\right)^{2}+A(t) \psi_{L}(r) \cos \alpha
$$

In writing down the critical layer equations, the instantaneous surface $r_{s}(\alpha, t)$, defined by $\partial_{r} \psi\left[r_{s}(\alpha, t)\right]=0$, is important as the location where dynamical equations turn singular and non-ideal effects come into play. As mode couplings do not affect the second order $m=1$ dynamics, one can keep the linear $m=1$ radial structure but introduce the corrections due to the motion of the $\partial_{r} \psi=0$ surface. This yields a differential equation $[4,5]$ for the $m=1$ amplitude $A(t)$ valid below the onset of "truly nonlinear" cubic nonlinearities on $m=1$. This is given by

$$
\frac{d A}{d t}=\gamma(t) A(t)
$$


with

$$
\frac{\gamma(t)}{\left\langle\frac{\psi_{0}^{\prime \prime}\left[r_{s}(\alpha, t)\right]}{r_{s}(\alpha, t)}\right\rangle_{\alpha}}=\frac{\gamma(t=0)}{\left\langle\frac{\psi_{0}^{\prime \prime}\left[r_{s}(\alpha, t=0)\right]}{r_{s}(\alpha, t=0)}\right\rangle_{\alpha}}=\frac{\gamma_{L}}{\frac{\psi_{0}^{\prime \prime}\left(r_{s 0}\right)}{r_{s 0}}},
$$

where $\langle\cdot\rangle_{\alpha} \equiv(2 \pi)^{-1} \int_{0}^{2 \pi} \cdot d \alpha$ denotes the $m=0$ average. As shown in Refs. [4,5], the differential equation (4), with $\gamma(t)$ given by (5), may actually be approximated by the quadratic expression

$$
\frac{d A}{d t}=\gamma_{L} A(t)-C_{0} A^{2}(t)
$$

with

$$
C_{0}=\frac{q^{\prime}\left(r_{s 0}\right)+r_{s 0} q^{\prime \prime}\left(r_{s 0}\right)-2 r_{s 0} q^{\prime}\left(r_{s 0}\right)^{2}}{\sqrt{2} \pi r_{s 0}^{2} q^{\prime}\left(r_{s 0}\right)} .
$$

It may be useful to remind here that the problem has been rendered dimensionless. The solution of (6) is

$$
A(t)=\frac{A_{0} \exp \left(\gamma_{L} t\right)}{1+A_{0} C_{0} / \gamma_{L}\left[\exp \left(\gamma_{L} t\right)-1\right]}
$$

that tends to $\gamma_{L} / C_{0}$ as $t \rightarrow \infty$. It is interesting to note that the curve $A / A_{0}$ has a universal form depending only on the rescaled time $\gamma_{L} t$ and on the parameter $A_{0} C_{0} / \gamma_{L}$ containing the $q$-profile properties at $r_{s 0}$. It is also interesting to note that, at time $t_{i}=\gamma_{L}^{-1} \ln \left[\gamma_{L} /\left(A_{0} C_{0}\right)-1\right], A(t)$ possesses an inflexion point so that, around that time, the effective behavior of $A$ is approximately algebraic (being linear). All this assumes that $C_{0}$ is positive. A negative $C_{0}$ would yield a transient explosive faster-than-exponential behavior. However, the validity of (6) is limited because some cubic nonlinearities should come into play. For $S$ not too large, so that the instantaneous second order location of the critical layer has some large overlap with the initial linear critical layer at $r_{s 0}$ around the X-point, it is possible $[4,5]$ that those cubic terms show up in a spectacular manner. When collecting terms cubic in $A$, it turns out that in this overlap domain the convective derivative due to the motion of the critical layer dominates the ordinary time derivative and equilibrates mode coupling terms yielding, together with (6), an effective amplitude equation of the form

$$
\frac{d A}{d t}-\gamma_{L} A+C_{0} A^{2}+\frac{c}{\eta} A^{2}\left(\frac{d A}{d t}-\gamma_{N L} A\right)=0
$$

with $c=\mathcal{O}(1)$ some constant and $\gamma_{N L}$ the growth rate reached by the $m=1$ 


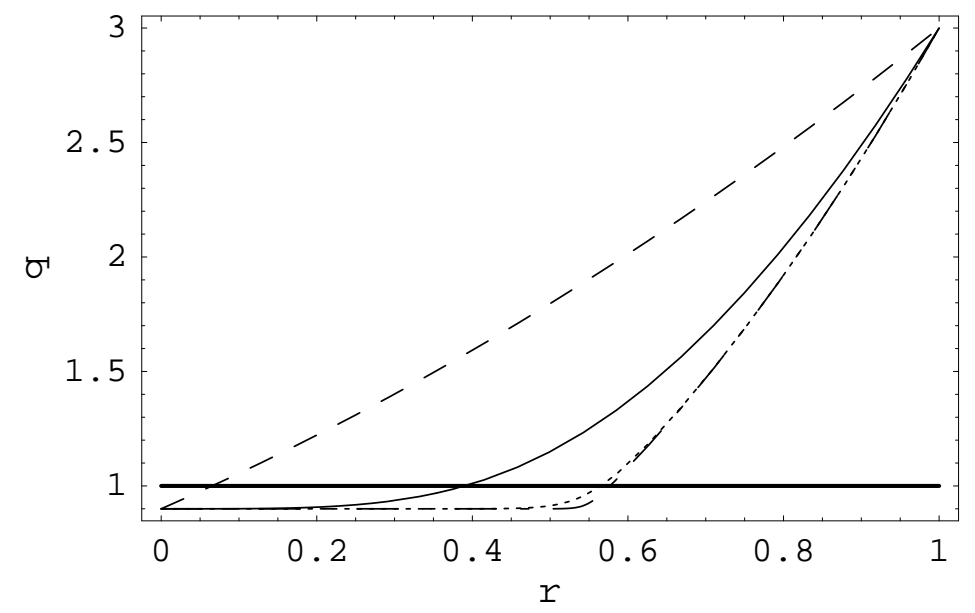

Fig. 1. Different $q$-profiles of the family $q(r)=q_{0}\left\{1+r^{2 \lambda}\left[\left(q_{a} / q_{0}\right)^{\lambda}-1\right]\right\}^{1 / \lambda}$ with $q_{0}=0.9$ and $q_{a}=3$ for $\lambda=0.6$ (dashed line), $\lambda=2$ (plain), $\lambda=10$ (dots) and $\lambda=30$ (dot-dashed line). The bold line is the $q=1$ threshold.

mode at the onset of cubic terms. This takes place for $A \sim \eta^{1 / 2}$, so that $\gamma_{N L} \sim \gamma_{L} \eta^{1 / 2}\left(1-\frac{C_{0}}{\gamma_{L}} \eta^{1 / 2}\right)$.

Let us finally investigate the influence of the $q$-profile in the onset of the nonlinear regime. The $q$-profiles $q(r)=q_{0}\left\{1+r^{2 \lambda}\left[\left(q_{a} / q_{0}\right)^{\lambda}-1\right]\right\}^{1 / \lambda}$, parameterized by $\lambda$, can depict different possible experimental situations (see Figure 1). The low $\lambda$ case is consistent with a very peaked current profile. Such a kind of profile was used for instance by Biskamp in his 1991's simulations [9] of the same system. He observed a transition from the linear exponential $m=1$ growth towards an algebraic behavior. On the contrary, the large $\lambda$ case coincides with a low shear situation with a flat current profile within the $q=1$ radius. This is reminiscent of recent experimental investigations undertaken e.g. in JET under the "hybrid" scenario, with a wide area of low magnetic shear and central safety factor close to and below one. Buratti and coworkers have reported in this case the wide emergence of "slow sawteeth" $[10,11]$ where the mode has the same spatial structure as the kink-like sawtooth precursor with $n=1$ but grows very slowly and enters the nonlinear regime at the linear growth rate. Although this case may not strictly correspond to the purely resistive mode, the present analysis on the onset of nonlinear effects should be transposable. Figure 2 may indeed propose an explanation for these observations. In the case of a very peaked $q$-profile (e.g. with $\lambda=0.6$ ), the integration of $m=1$ amplitude evolution Eq. (8) before the onset of third order convective effects shows that $A$ should saturate before this third order threshold $A \sim \eta^{1 / 2}$ is reached. In particular, this may explain Biskamp's observations [9] of a transition to an algebraic stage with no subsequent nonlinear exponential growth. The $\lambda=2$ case just corresponds to the $q$-profile taken by Aydemir in Ref. [2]. Here the third order convective stage showing a second stage of exponential growth can be reached. It is clear from Fig. 2 that the nonlinear growth rate 


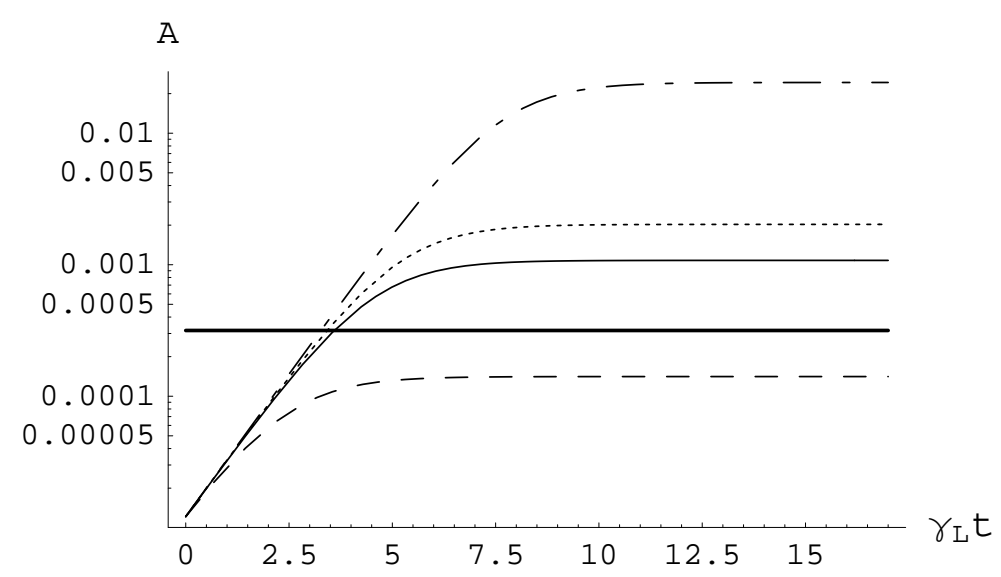

Fig. 2. Evolution (in linear-log scale) of the amplitude of the $m=1$ mode, given by Eq. (8), as a function of time normalized to the linear growth rate for the $q$-profiles displayed in Fig. 1 (with the same plot styles) before the onset of "third order" nonlinear regime. The bold horizontal line marks the threshold of third order convective terms for $A \sim S^{-1 / 2}$ for the value $S=10^{7}$. The initial amplitude is $A_{0}=1.2 \times 10^{-5}$.

$\gamma_{N L}$, that is the growth rate of the $m=1$ mode when $A$ crosses the threshold $\left(A \sim \eta^{1 / 2}\right)$ is (slightly) smaller than $\gamma_{L}$ as $A$ has turned bending. Indeed the simulations of Ref. [2] give the numerical value $\gamma_{N L} \simeq \gamma_{L} / 2$. Yet, for larger values of $\lambda$, corresponding to a $q$-profile of the kind studied by Buratti et al. $[10,11]$, it is clear from Figure 2 that $\gamma_{N L}$ would be almost equal to $\gamma_{L}$ : This is an explanation of the fact that, in "slow sawteeth", the $m=1$ mode enters the nonlinear regime at almost the linear growth rate.

\section{Acknowledgements}

The author is greatly indebted to B. Coppi for his advice and explanations and thanks the organizers and participants of the 9th Plasma Easter Meeting held in Torino for the nice meeting.

\section{References}

[1] B. Coppi, R. Galvão, M.N. Rosenbluth and P.H. Rutherford, Soviet Journal of Plasma Physics 2, 3276 (1976).

[2] A.Y. Aydemir, Phys. Rev. Lett. 78, 4407 (1997).

[3] B.V. Waddell, M.N. Rosenbluth, D.A. Monticello and R.B. White, Nucl. Fusion 16, 3 (1976). 
[4] M.C. Firpo, Phys. Plasmas 11, 970 (2004).

[5] M.C. Firpo and B. Coppi, Phys. Rev. Lett. 90, 095003 (2003).

[6] R. Fitzpatrick, Plasma Phys. Control. Fusion 31, 1127 (1989).

[7] D.F. Escande and M. Ottaviani, Physics Letters A 323, 278 (2004).

[8] F. Militello and F. Porcelli, Physics of Plasmas 11, L13 (2004).

[9] D. Biskamp, Phys. Fluids B 3, 3353 (1991).

[10] P. Buratti, B. Alper, A. Becoulet, P. Belo, C. Gormezano, P. Smeulders and EFDA-JET Contributors, 31st EPS Conference on Plasma Physics, London, Europhysics Conference Abstracts 28G, P.1-165 (2004).

[11] C. Gormezano, A. Becoulet, P. Buratti et al., Plasma Physics and Controlled Fusion 46, B435 (2004). 